\title{
AN ALTERNATIVE CONDITION FOR STOCHASTIC DOMINATION
}

\author{
KENSHI HOSAKA, ${ }^{*}$ Ritsumeikan University
}

\begin{abstract}
We will propose an alternative condition for stochastic domination. This condition differs in an essential way from the strong likelihood ratio property. We also show an example which satisfies the new condition, but does not satisfy the strong likelihood ratio property.
\end{abstract}

Keywords: Strong likelihood ratio property; stochastic domination

2000 Mathematics Subject Classification: Primary 60A10

Secondary 60C05

\section{Introduction}

Let $E$ be a finite set and, for each $e \in E$, let $\left(\Omega_{e}, \mathcal{F}_{e}, \mu_{e}\right)$ be a measure space with $\mu_{e}$ a nonnegative $\sigma$-finite measure. Suppose that $\Omega_{e}$ is equipped with a total order ' $\geq$ ' that is $\mathcal{F}_{t}$-measurable. We take as the configuration space the set $\Omega=\prod_{e \in E} \Omega_{e}$ and the corresponding product $\sigma$-algebra $\mathcal{F}=\prod_{e \in E} \mathcal{F}_{e}$, and we let $\mu=\prod_{e \in E} \mu_{e}$. The set $\Omega$ is a partially ordered set with partial order given by $\omega_{1} \leq \omega_{2}$ if and only if $\omega_{1}(e) \leq \omega_{2}(e)$ for all $e \in E$. A random variable $X: \Omega \rightarrow \mathbb{R}$ is called increasing if $X\left(\omega_{1}\right) \leq X\left(\omega_{2}\right)$ whenever $\omega_{1} \leq \omega_{2}$ (see [1,p. 23] or [2, p. 19]). Preston [6] showed that if a pair of probability densities satisfies the condition

$$
f_{2}\left(\omega_{1} \vee \omega_{2}\right) f_{1}\left(\omega_{1} \wedge \omega_{2}\right) \geq f_{1}\left(\omega_{1}\right) f_{2}\left(\omega_{2}\right), \quad \omega_{1}, \omega_{2} \in \Omega,
$$

where $\omega_{1} \vee \omega_{2}=\left(\omega_{1}(e) \vee \omega_{2}(e): e \in E\right), \omega_{1}(e) \vee \omega_{2}(e)=\max \left\{\omega_{1}(e), \omega_{2}(e)\right\}, \omega_{1} \wedge \omega_{2}=$ $\left(\omega_{1}(e) \wedge \omega_{2}(e): e \in E\right)$, and $\omega_{1}(e) \wedge \omega_{2}(e)=\min \left\{\omega_{1}(e), \omega_{2}(e)\right\}$, then, for any increasing random variable $X$ on $\Omega$,

$$
\int X(\omega) f_{2}(\omega) \mathrm{d} \mu(\omega) \geq \int X(\omega) f_{1}(\omega) \mathrm{d} \mu(\omega) .
$$

We say that $f_{1}$ is stochastically dominated by $f_{2}$, written $f_{2} \succ_{\mathfrak{D}} f_{1}$ (see [1, p. 23]). We call condition (1.1) the strong likelihood ratio property (see [3] or [4, p. 129]). We also use the following notation for (1.1):

$$
f_{2} \succ_{T P_{2}} f_{1} \text {. }
$$

In this paper we show an alternative sufficient condition for stochastic domination. However, this sufficient condition is deduced from the strong likelihood ratio property. In Section 2 we give its exact statement. In Section 3 we give a proof of this statement. In Section 4 we give two examples. The first example satisfies our new sufficient condition (and, therefore, satisfies stochastic domination), but it does not satisfy the strong likelihood ratio property (1.1). On the other hand, the second example satisfies the strong likelihood ratio property, but it does not satisfy our new sufficient condition. This example shows that our sufficient condition does not include the strong likelihood ratio property.

Received 1 June 2009; revision received 26 August 2009.

* Postal address: 1-1-1 Noji Higashi, Kusatsu, Shiga, Japan. Email address: khosaka@fc.ritsumei.ac.jp 


\section{Statement of the result}

In this section we give an alternative sufficient condition.

Definition 2.1. Let $f_{1}$ and $f_{2}$ be probability densities on $\Omega$. We say that $f_{2}$ is larger than $f_{1}$ in the likelihood difference order, written $f_{2} \succ_{\text {ld }} f_{1}$, if $f_{2}-f_{1}$ is increasing on $\Omega$.

Remark 2.1. A binary relation ' $\succ_{\text {ld }}$ ' is a partial order on the set of all probability densities on $\Omega$.

Theorem 2.1. Let $f_{1}$ and $f_{2}$ be probability densities on $\Omega$ which satisfy $f_{2} \succ_{\operatorname{ld}} f_{1}$. Then $f_{2} \succ_{D} f_{1}$.

Remark 2.2. Definition 2.1 gives a new sufficient condition for stochastic domination (see Example 4.1, below). However, Definition 2.1 is not implied by the strong likelihood ratio property (see Example 4.2, below). Therefore, Definition 2.1 is not a necessary condition for stochastic domination.

\section{Proof of Theorem 2.1}

In this section we give a proof of Theorem 2.1 using the Preston theorem. First, we shall prepare the following notation to prove Theorem 2.1. Let $f$ be a function on $\Omega$ given by

$$
f(\omega)=f_{2}(\omega)-f_{1}(\omega),
$$

satisfying $\int f(\omega) \mathrm{d} \mu(\omega)=0$. Note that $f$ is increasing. Let $P$ and $N$ be measurable sets of $\Omega$ such that

$$
P=\{\omega \in \Omega: f(\omega) \geq 0\}, \quad N=\{\omega \in \Omega: f(\omega)<0\} .
$$

We remark that

$$
\int_{P} f(\omega) \mathrm{d} \mu(\omega)=-\int_{N} f(\omega) \mathrm{d} \mu(\omega) \equiv M .
$$

We define probability densities $f_{+}$and $f_{-}$as follows:

$$
f_{+}(\omega)=\frac{f(\omega) \vee 0}{M}, \quad \omega \in \Omega, \quad f_{-}(\omega)=\frac{-f(\omega) \vee 0}{M}, \quad \omega \in \Omega .
$$

We note that $f_{+}$is increasing and $f_{-}$is decreasing. Therefore, the pair $f_{+}$and $f_{-}$satisfies the strong likelihood ratio property

$$
f_{+}\left(\omega_{1} \vee \omega_{2}\right) f_{-}\left(\omega_{1} \wedge \omega_{2}\right) \geq f_{-}\left(\omega_{1}\right) f_{+}\left(\omega_{2}\right), \quad \omega_{1}, \omega_{2} \in \Omega .
$$

Then, by the Preston theorem [6], we obtain

$$
\int X(\omega) f_{+}(\omega) \mathrm{d} \mu(\omega) \geq \int X(\omega) f_{-}(X) \mathrm{d} \mu(\omega)
$$

for an arbitrary increasing function $X$. So, we have

$$
\begin{aligned}
\int X & (\omega) f_{2}(\omega) \mathrm{d} \mu(\omega)-\int X(\omega) f_{1}(\omega) \mathrm{d} \mu(\omega) \\
& =\int X(\omega) f(\omega) \mathrm{d} \mu(\omega) \\
& =M\left\{\int X(\omega) f_{+}(\omega) \mathrm{d} \mu(\omega)-\int X(\omega) f_{-}(\omega) \mathrm{d} \mu(\omega)\right\} \\
& \geq 0 .
\end{aligned}
$$




\section{Example}

In this section we provide two examples. The first example does not satisfy the strong likelihood ratio property (1.1), but does satisfy the condition of Definition 2.1. The second example is opposite to the first example; it satisfies the strong likelihood ratio property, but does not satisfy Definition 2.1.

Example 4.1. Let $\mu$ be the uniform measure on $\Omega=\{0,1\}^{3}$. Let $f_{1}$ and $f_{2}$ be probability densities defined by

$$
\begin{aligned}
& f_{2}(111)=\frac{1}{8}, \\
& f_{2}(000)=\frac{1}{8}-\delta, \\
& f_{2}(110)=f_{2}(101)=f_{2}(011)=\frac{1}{8}+\frac{\delta}{3}, \\
& f_{2}(100)=f_{2}(010)=f_{2}(001)=\frac{1}{8},
\end{aligned}
$$

for $\frac{1}{8} \geq \delta>0$, and define $f_{1}$ symmetrically by $f_{1}(x y z)=f_{2}([1-x][1-y][1-z])$.

Claim 4.1. The pair $f_{1}$ and $f_{2}$ satisfies $f_{2} \succ_{\mathrm{ld}} f_{1}$; however, $f_{1} \nsucc_{T P_{2}} f_{2}$.

Proof. It is easy to check the required properties of $f_{1}$ and $f_{2}$. For example, if we take $\omega_{1}=(001)$ and $\omega_{2}=(110)$, then we have the following inequality:

$$
f_{2}\left(\omega_{1} \vee \omega_{2}\right) f_{1}\left(\omega_{1} \wedge \omega_{2}\right)<f_{1}\left(\omega_{1}\right) f_{2}\left(\omega_{2}\right) .
$$

Example 4.2. Nagahata [5] pointed out the following example. Let $\Omega=\{0,1\}^{2}$ and $\mu$ be the uniform measure. Let $p$ and $q$ satisfy $\frac{1}{4} \geq p>q \geq 0$. We consider a couple of product Bernoulli densities $P_{p}$ and $P_{q}$. This pair satisfies $P_{p} \succ_{T P_{2}} P_{q}$; however, $P_{p} \nsucc_{\text {ld }} P_{q}$. It is easy to check this.

\section{Acknowledgements}

I am most grateful to Professor Geoffrey R. Grimmett for giving me valuable comments and encouragement. I am also grateful to Professor J. van den Berg, Professor Y. Nagahata, Professor N. Sugimine, and Professor Y. Higuchi for giving me important comments. Finally, I would like to thank the anonymous referee for giving me valuable advice.

\section{References}

[1] Georgit, H.-O., Häggström, O. And Maes, C. (2000). The random geometry of equilibrium phases. In Phase Transitions and Critical Phenomena, Vol. 18, eds C. Domb and J. L. Lebowitz, Academic Press, London, pp. 1142 .

[2] Grimmett, G. (2006). The Random-Cluster Model. Springer, Berlin.

[3] Karlin, S. And RinotT, Y. (1980). Classes of orderings of measures and related correlation inequalities. I. Multivariate totally positive distributions. J. Multivariate Anal. 10, 467-498.

[4] Müller, A. And Stoyan, D. (2002). Comparison Methods for Stochastic Models and Risks. John Wiley, Chichester.

[5] Nagahata, Y. (2006). Private communication.

[6] Preston, C. J. (1974). A generalization of the FKG inequalities. Commun. Math. Phys. 36, 233-241. 